\title{
Expression and significance of the novel tumor-suppressor gene SMG-1 in hepatocellular carcinoma
}

\author{
LI-LI HAN $^{1 *}$, HAO-CHENG NAN ${ }^{2 *}$, TAO TIAN ${ }^{1}$, HUI GUO ${ }^{1}$, TING-HUA HU ${ }^{1}$, WEN-JUAN WANG ${ }^{1}$, \\ JIE-QUN MA ${ }^{1}$, LI-LI JIANG ${ }^{1}$, QIAN-QIAN GUO ${ }^{1}$, CHENG-CHENG YANG ${ }^{1}$, \\ XIAO-MIN KANG ${ }^{1}$, YING LIU ${ }^{1}$, YUAN GAO ${ }^{1}$, QI-LUN LIU ${ }^{2}$ and KE-JUN NAN ${ }^{1}$ \\ ${ }^{1}$ Department of Oncology, First Affiliated Hospital, College of Medicine of Xi'an Jiaotong University, \\ Xi'an, Shaanxi 710061; ${ }^{2}$ Department of Surgical Oncology, General Hospital of Ningxia Medical University, \\ Yinchuan, Ningxia Hui Autonomous Region 750004, P.R. China
}

Received February 7, 2014; Accepted March 20, 2014

DOI: 10.3892/or.2014.3125

\begin{abstract}
Recent studies have demonstrated that SMG-1, a newly characterized member of the family of phosphatidylinositol 3-kinase-related protein kinases (PIKKs), is involved in tumorigenesis as a new tumor suppressor. However, its expression and significance in hepatocellularcarcinoma (HCC) remain obscure. The present study investigated SMG-1 expression in HCC tissue specimens, aimed at defining the association with clinicopathological significance. Both immunohistochemistry and qRT-PCR were employed to analyze SMG-1 expression in $157 \mathrm{HCC}$ and corresponding distant normal tissue specimens. The results revealed that expression of SMG-1 was significantly lower in the HCC tissue specimens than that in the distant normal tissues. Moreover, a lower expression level of SMG-1 was significantly correlated with serum $\alpha$-fetoprotein level $(\mathrm{P}=0.001)$, poorly differentiated tumors $(\mathrm{P}=0.009)$ and more advanced TNM stage $(\mathrm{P}<0.001)$. Further study showed that SMG-1 expression was exactly associated with tumor differentiation and clinical stage in HCC. Kaplan-Meier analysis indicated that low SMG-1 expression was related to poor overall survival, and the prognostic impact of SMG-1 was further confirmed by stratified survival analysis. Importantly, multivariate analysis revealed that low SMG-1 expression was an independent prognostic marker for an unfavorable overall survival. We conclude that SMG-1 is downregulated in HCC
\end{abstract}

Correspondence to: Professor Qi-Lun Liu, Department of Surgical Oncology, General Hospital of Ningxia Medical University, Yinchuan City, Ningxia Hui Autonomous Region 750004, P.R. China E-mail: liuql16311@hotmail.com

Professor Ke-Jun Nan, Department of Oncology, First Affiliated Hospital, College of Medical of Xi'an Jiaotong University, Xi'an, Shaanxi 710061, P.R. China

E-mail:nankj@163.com

${ }^{*}$ Contributed equally

Key words: SMG-1, hepatocellular carcinoma, biomarker and may represent a promising biomarker for predicting the prognosis of $\mathrm{HCC}$, including the prognosis of early-stage patients.

\section{Introduction}

Hepatocellular carcinoma (HCC) is the second leading cause of cancer-related mortality in men and the sixth leading cause of cancer-related mortality in women and is the most frequently diagnosed cancer worldwide (1). In addition, HCC is one of the deadliest primary cancers, and its 5-year survival rate is $10 \%$ or less (2). Limited treatment options and late diagnosis can explain the low survival rate (3). The discovery of more satisfactory molecular biomarkers could significantly improve the survival rate of $\mathrm{HCC}$ as early diagnosis and prediction help to assess tumor metastasis, tumor advancement and evaluate therapeutic strategies (4).

HCC carcinogenesis involves aberrant changes in multiple molecular pathways and genetic alterations, which consequently result in malignant transformation and progression of HCC (5). Each step of the signaling pathways involved in the pathogenesis of HCC could be a target by which to inhibit HCC progression. Several novel molecularly targeted drugs are currently under preclinical and clinical trials for the treatment of HCC. Yet, to date, only sorafenib has been found to prolong the survival of patients with advanced HCC (6). The effects of the majority of targeted drug treatments remain unsatisfactory. In conclusion, it is of great clinical value to further identify effective biomarkers of HCC not only for diagnosis and prognosis but also for use as novel therapeutic targets.

Suppressor with morphogenetic effect on genitalia (SMG-1) is the most recently characterized member of the family of phosphatidylinositol 3-kinase-related protein kinases (PIKKs). It plays a central role in cell growth and stress response pathways, and is conserved across species. SMG-1 is involved in a mechanism responsible for the degradation of premature stop codon containing mRNA, also called NMD (nonsense mediated mRNA decay) in C. elegans and in mammalian cells (7-9). In addition to NMD, SMG-1 has been shown to play other cellular roles, such as regulation of the G1/S checkpoint during oxidative stress (10), protection 
against $\mathrm{TNF} \alpha$-dependent apoptosis (11), response to hypoxia, IR and UV radiation $(12,13)$, as well as lifespan regulation in Caenorhabditis elegans (14), and a novel regulator of regeneration and animal growth with an antagonistic role to mTOR signaling in planarians (15).

Furthermore, recent studies have demonstrated that SMG-1 is also involved in tumorigenesis. Gubanova et al (16) reported that SMG-1 suppresses tumor growth via regulation of both the p53 and Cdc25A signaling pathways. Similar findings in another study using a SMG-1-knockout mouse model which predisposed to a whole range of tumor formation and inflammation suggested that SMG-1 is a tumor suppressor (17).

It is well known that deregulation of cell cycle control plays an important role in the multistep process of hepatocarcinogenesis. The signaling pathways involved in regulation of the cell cycle from the G1 phase to the $S$ phase are frequent targets of carcinogenic events. A key regulator of G1/S arrest is SMG-1. SMG-1 has been shown to control G1/S cell cycle arrest through both the p53-dependent and the p53-independent pathways (16). Yet, no report exists regarding the mRNA and protein expression levels of SMG-1 in human HCC, and whether SMG-1 is an oncogene or a tumor-suppressor gene in HCC has not yet been demonstrated.

To clarify the role of SMG-1 in HCC, we investigated the mRNA and protein expression of SMG-1 in different pathological subtypes of primary HCC using real-time PCR assay and immunohistochemistry. The relationship between expression of SMG-1 and clinicopathological parameters, and patient survival in primary HCC was analysed. We showed abnormal expression of SMG-1 in HCC tissues compared to the corresponding distant normal tissues. SMG-1 may be a key biomarker of HCC tumors and patient prognosis. In conclusion, low expression of SMG-1 is closely related to progression of HCC, and may be regarded as a predictor of poor prognosis of HCC.

\section{Materials and methods}

Liver cancer tissue specimens. Formalin-fixed, paraffinembedded primary HCC specimens obtained from 157 patients, who received surgical resection without prior radiotherapy or chemotherapy between August 2007 and December 2009 were randomly selected from the archives of The First Affiliated Hospital of Medical College of Xi'an Jiaotong University. The fresh HCC tissue specimens, together with their paired adjacent non-cancerous tissues (5-cm from the cancer region) from each patient, were obtained during the surgical operation, snap frozen in liquid nitrogen and subsequently stored at $-80^{\circ} \mathrm{C}$ until use for experimental purposes. For the use of these clinical materials for research purposes, prior patient written informed consent and approval from the Institutional Research Ethics Committee of the Medical College of Xi'an Jiaotong University and its First Affiliated Hospital were obtained. Histological diagnosis was performed for all of the cases by three independent experienced pathologists. The cohort of patients with HCC included 137 (87.3\%) men and 20 (12.7\%) women. The mean age was 48.7 years, with ages ranging from 19 to 79 years, and the patient clinicopathological data are summarized in Table I. Tumor stages were assigned according to the 2002 American Joint Committee on Cancer/International
Union against Cancer Tumor/Lymph Node Metastasis/Distal Metastasis (TNM) classification system. Tumor cellular differentiation was assessed using Edmondson's classification. Hepatitis B virus (HBV) infection was diagnosed when HBV surface antigen (HBsAg) was detected by ELISA in the serum. The follow-up for all of the cases was completed in August of 2013. The cases lost to follow-up and those ending in death from causes other than HCC were regarded as censored data during the survival analysis.

Immunohistochemistry. Semi-quantitative immunohistochemistry (IHC) was used to determine the SMG-1 protein levels. Briefly, after deparaffinization and re-hydration of tissue sections, the sections were first subjected to antigen retrieval in a pressure cooker in citric buffer for $10 \mathrm{~min}$. The sections were then incubated with $3 \% \mathrm{H}_{2} \mathrm{O}_{2}$ for $10 \mathrm{~min}$ at room temperature to block potential endogenous peroxidase activity and then incubated with $20 \%$ normal serum and further with a primary antibody against SMG-1 (sc-135563, 1:100; Santa Cruz Biotechnology, Inc., Santa Cruz, CA, USA) at $4^{\circ} \mathrm{C}$ overnight. The negative control sections were incubated with phosphatebuffered saline (PBS) only to replace the primary antibody. The next day the sections were washed thrice with PBS and then incubated with a biotinylated secondary antibody for $30 \mathrm{~min}$ at room temperature. Diaminobenzidine substrate was used to reveal immunoreactive products in the sections. After counterstaining with hematoxylin to reveal nuclei, the sections were mounted on slides and coverslipped. To assess immunopositive cells, 3 pathologists reviewed the immunostained sections under a light microscope and scored the sections in 10 random $\mathrm{x} 20$ power fields. The staining intensity was graded as '0' (negative staining), ' 1 ' (weak staining), '2' (moderate staining) and ' 3 ' (strong staining). The percentage of positively stained cells was scored as: '0' (0\%), '1' (1-25\%), '2' (26-50\%), ' 3 ' (51-75\%) and '4' (76-100\%). These 2 scores were added together. Based on the sum of the scores, each tissue sample was categorized into 4 groups: $0, \leq 5 \%$ cells were stained; $1-3$, weak expression; 4-5, moderate expression; and 6-7, strong expression. Finally, we compared statistically the numbers of cells with low-to-weak expression with those with moderateto-strong expression. Furthermore, 10 representative staining fields of each section were analyzed to verify the mean optical density (MOD), which represented the strengths of staining signals as measured per positive pixels. The MOD data were statistically analyzed using t-test to compare the average MOD difference between different groups of tissues, and $\mathrm{P}<0.05$ was considered to indicate a statistically significant result.

\section{Real-time reverse transcriptase-polymerase chain reaction} (qRT-PCR). Total mRNA was extracted from tissue samples using TRIzol reagent (Invitrogen-Life Technologies, Carlsbad, CA, USA) and reverse-transcribed into cDNA using an RT-PCR kit (Takara, Dalian, China). Amplification of these cDNA samples was performed using SYBR Premix Ex Taq ${ }^{\mathrm{TM}} \mathrm{II}$ (Takara) in an iQ5 Multicolor real-time PCR detection system (Bio-Rad Laboratories, Hercules, CA, USA). The primers for SMG-1 and glyceraldehyde-3-phosphate dehydrogenase (GAPDH; internal control) were designed using Primer Express v2.0 software (Applied Biosystems) and are provided as follows: SMG-1 (forward: 5'-GGTGGCTCGATGTTAC 
Table I. Correlation between the clinicopathological variables and SMG-1 expression in the HCC cases $(n=157)$.

\begin{tabular}{|c|c|c|c|c|c|}
\hline \multirow[b]{2}{*}{ Variable } & \multirow[b]{2}{*}{ All cases, $\mathrm{n}(\%)$} & \multicolumn{2}{|c|}{ SMG-1 protein } & \multirow[b]{2}{*}{$\chi^{2}$} & \multirow[b]{2}{*}{ P-value ${ }^{a}$} \\
\hline & & Low expression, $\mathrm{n}(\%)$ & High expression, n (\%) & & \\
\hline Age $\left(\right.$ years) ${ }^{b}$ & & & & 0.149 & 0.700 \\
\hline$<48.7$ & 65 (41.4) & $45(69.2)$ & $20(30.8)$ & & \\
\hline$\geq 48.7$ & $92(58.6)$ & $61(66.3)$ & $31(33.7)$ & & \\
\hline Gender & & & & 0.001 & 0.976 \\
\hline Female & $31(19.7)$ & $21(67.7)$ & $10(32.3)$ & & \\
\hline Male & $126(80.3)$ & $85(67.5)$ & $41(32.5)$ & & \\
\hline HBsAg & & & & 0.064 & 0.800 \\
\hline Positive & $137(87.3)$ & $92(67.2)$ & $45(32.8)$ & & \\
\hline Negative & $20(12.7)$ & $14(70.0)$ & $6(30.0)$ & & \\
\hline $\operatorname{AFP}(\mathrm{ng} / \mathrm{ml})$ & & & & 12.073 & 0.001 \\
\hline$<400$ & $99(63.0)$ & $57(57.6)$ & $42(42)$. & & \\
\hline$\geq 400$ & $58(37.0)$ & $49(84.5)$ & $9(15.5)$ & & \\
\hline Cirrhosis & & & & 0.005 & 0.946 \\
\hline Yes & $101(64.3)$ & $68(67.3)$ & $33(32.7)$ & & \\
\hline No & $56(35.9)$ & $38(67.9)$ & $18(32.1)$ & & \\
\hline Tumor size $(\mathrm{cm})$ & & & & 0.432 & 0.511 \\
\hline$<5$ & $68(43.3)$ & $44(64.7)$ & $24(35.3)$ & & \\
\hline$\geq 5$ & $89(56.7)$ & $62(69.7)$ & $27(30.3)$ & & \\
\hline Tumor multiplicity & & & & 1.239 & 0.266 \\
\hline Single & $93(59.2)$ & $66(71.0)$ & $27(29.0)$ & & \\
\hline Multiple & $64(40.7)$ & $40(62.5)$ & $24(37.5)$ & & \\
\hline Differentiation & & & & 6.777 & 0.009 \\
\hline Well-moderate & $72(45.9)$ & $41(56.9)$ & $31(43.1)$ & & \\
\hline Poor-undifferentiated & $85(54.1)$ & $65(76.5)$ & $20(23.5)$ & & \\
\hline Stage & & & & 35.266 & 0.000 \\
\hline I-II & $67(42.7)$ & $28(41.8)$ & $39(58.2)$ & & \\
\hline III-IV & $90(57.3)$ & $78(86.7)$ & $12(13.3)$ & & \\
\hline Hepatic vein invasion & & & & 1.478 & 0.224 \\
\hline Yes & $47(30.0)$ & $35(74.5)$ & $12(25.5)$ & & \\
\hline No & $110(70.0)$ & $71(64.5)$ & $39(35.5)$ & & \\
\hline
\end{tabular}

CCTC-3' and reverse, 5'-CTGCGTGGCGAAGGTTTC-3'); GAPDH (forward, 5'-ACCACAGTCCATGCCATCAC-3' and reverse, 5'-TCCACCACCCTGTTGCTGTA-3'). Expression levels of SMG-1 were evaluated using a relative quantification approach ( $2^{-\Delta \Delta \mathrm{Ct}}$ method) against GAPDH levels.

Statistical analysis. Comparisons between groups for statistical significance were performed with a two-tailed paired Student's t-test. The Chi-square $\left(\chi^{2}\right)$ test was performed to analyze the correlation between SMG-1 expression and clinicopathological parameters. The Kaplan-Meier method (the log-rank test) was used for survival analysis and univariate analysis. The Cox proportional hazards regression model was used for the multivariate analysis of survival duration. Statistical Package for Social Science (SPSS) version 17.0 was used. All reported P-values were two-sided. P-values were considered statistically significant at the $\mathrm{P}<0.05$ level, and a value of $\mathrm{P}<0.01$ was considered to indicate a highly significant difference.

\section{Results}

Expression of SMG-1 $\mathrm{mRNA}$ and protein in hepatocellular carcinoma tissue samples by real-time PCR and immunohistochemistry. To examine the expression of SMG-1, 157 pairs of HCC and adjacent non-cancerous tissues were subjected to qRT-PCR. Results showed that the level of SMG-1 mRNA was significantly lower in the HCC tissues than the level in the distant non-cancerous tissues $(1.13 \pm 0.17$ compared with $3.82 \pm 0.29 ; \mathrm{t}=23.32 ; \mathrm{P}<0.01$ ) (Fig. 1). To further examine the expression of SMG-1 in HCC, we analyzed the expression of SMG-1 protein using IHC. Based on the results of IHC, low 


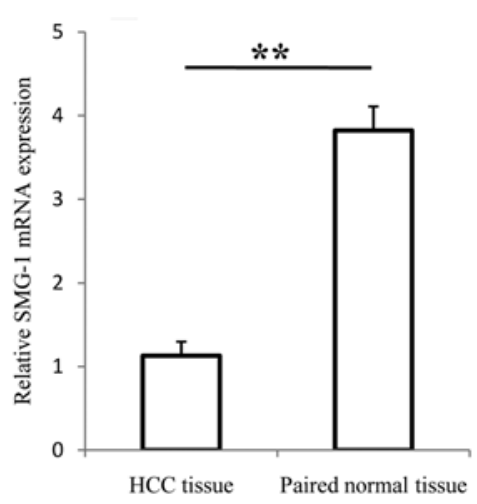

Figure 1. Relative expression of SMG-1 mRNA in HCC tissue and paired normal tissues was quantified by real-time PCR in 157 HCC patients. ${ }^{* *} \mathrm{P}<0.01$.

SMG-1 expression in tumor tissues was identified in $67.5 \%$ $(106 / 157)$ of the cases. Moreover, in $71.3 \%(112 / 157)$ of the HCC patients, less SMG-1 was expressed in the tumor tissues. IHC results confirmed the qRT-PCR data and indicated that SMG-1 was significantly reduced in the HCC tissues when compared with that in the distant non-cancerous tissues $\left(\chi^{2}=11.207, \mathrm{P}=0.001\right)$ (Fig. 2). These results indicate that SMG-1 may represent a biological marker of the presence of HCC in humans.

Association between SMG-1 expression and clinical variables of the hepatocellular carcinoma cases. To determine the clinical significance of SMG-1 in HCC, the correlation between expression of SMG-1 and clinicopathological features was analyzed. Significant associations were found with tumor differentiation $(\mathrm{P}=0.009)$, clinical stage $(\mathrm{P}<0.001)$ and serum
Table II. Univariate analysis of survival data.

\begin{tabular}{lc}
\hline Clinicopathological characteristics & P-value \\
\hline Age, $<48.7$ vs. $\geq 48.7$ years & 0.343 \\
Gender, male vs. female & 0.498 \\
AFP, $<400$ vs. $\geq 400 \mathrm{ng} / \mathrm{ml}$ & 0.001 \\
Hepatitis B virus infection, positive vs. negative & 0.539 \\
Tumor size, $<5$ vs. $\geq 5 \mathrm{~cm}$ & 0.006 \\
Histological grade, well/moderate vs. poor & 0.017 \\
TNM stage, I + II vs. III + IV & 0.000 \\
SMG-1, high expression vs. low expression & 0.001
\end{tabular}

AFP level $(\mathrm{P}=0.001)$, indicating that $\mathrm{HCC}$ in patients with low SMG-1 expression was frequently associated with a high level of serum AFP, poor tumor differentiation and advanced clinical stage (Table I). The significant correlation between SMG-1 expression in HCC and tumor differentiation was further confirmed. The percentage of cases with low SMG-1 expression was markedly higher in poorly differentiated HCC than that in well-differentiated HCC. Higher expression of SMG-1 was observed in well-differentiated HCC. Conversely, low SMG-1 expression was noticeably significant in poorly differentiated HCC (Fig. 3).

Furthermore, the IHC analysis showed that SMG-1 was weak or negative in HCC lesions of patients with late stages of HCC (TNM stages III-IV) as compared with that in early-stage HCC (TNM stages I-II) tissues $(\mathrm{P}<0.05$; Fig. 4).Quantitative analysis of the IHC staining indicated that SMG-1 expression in clinical stage III-IV primary tumors was statistically lower than that in clinical stage I-II primary tumors ( $\mathrm{P}<0.05$; Fig. 4$)$.
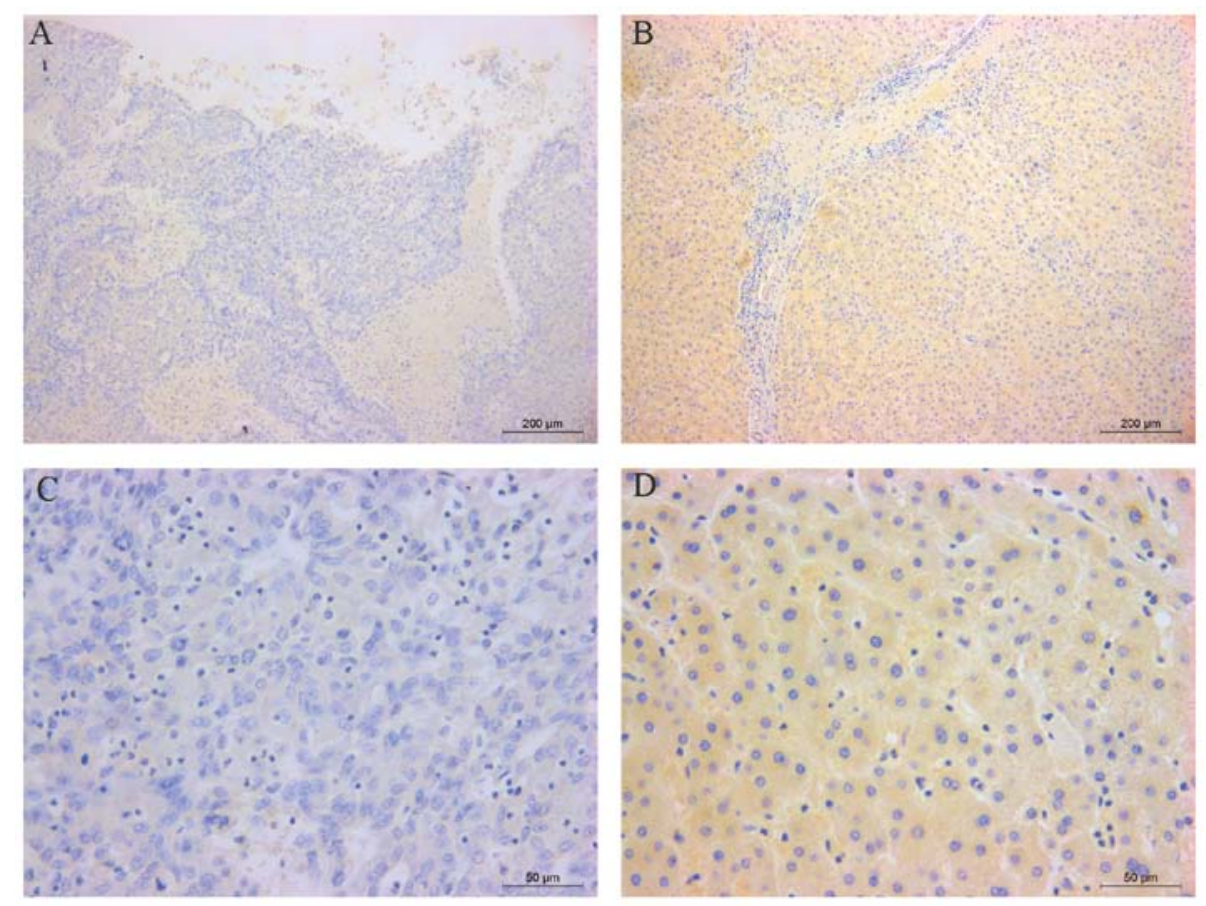

Figure 2. The level of SMG-1 protein in the HCC tissue and adjacent non-cancerous tissue samples obtained from the same patient using IHC. (A) Expression of SMG-1 in HCC tissues (original magnification, x100). (B) Expression of SMG-1 in adjacent non-tumor tissues (original magnification, x100). (C) Expression of SMG-1 in HCC tissues (original magnification, x400). (D) Expression of SMG-1 in adjacent non-tumor tissues (original magnification, x400). 


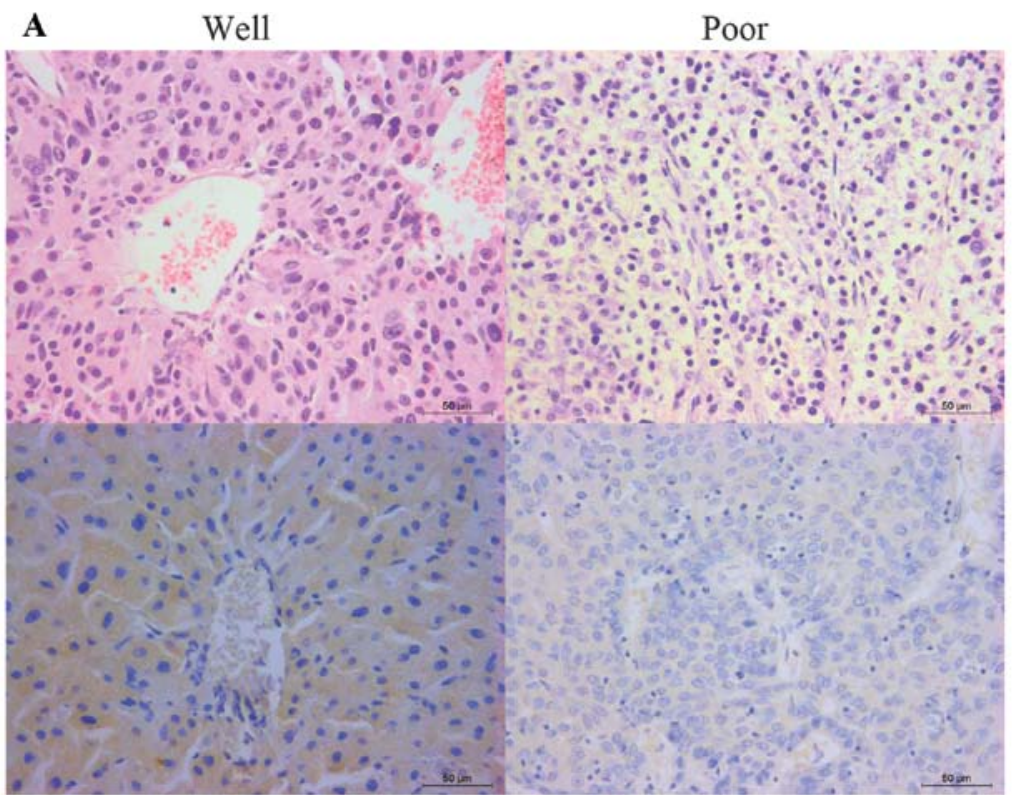

\section{$H \& E$}

SMG-1

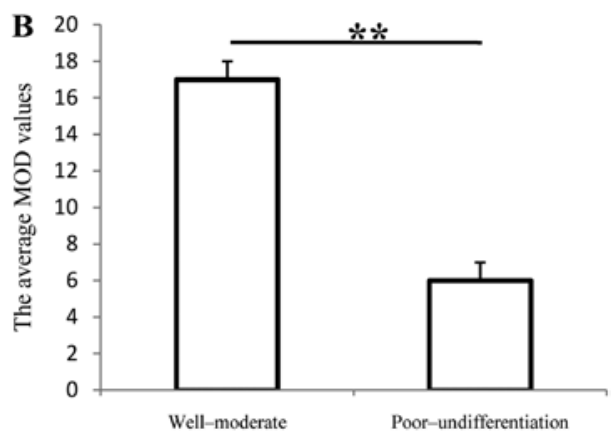

Figure 3. Expression of SMG-1 was lower in HCC with poor differentiation. (A) Representative micrographic images showing the SMG-1 expression in well-differentiated and poorly differentiated HCC. (B) A decreasing trend of SMG-1 expression was noted from well differentiated to poorly differentiated HCC. ${ }^{*} \mathrm{P}<0.05$.

A

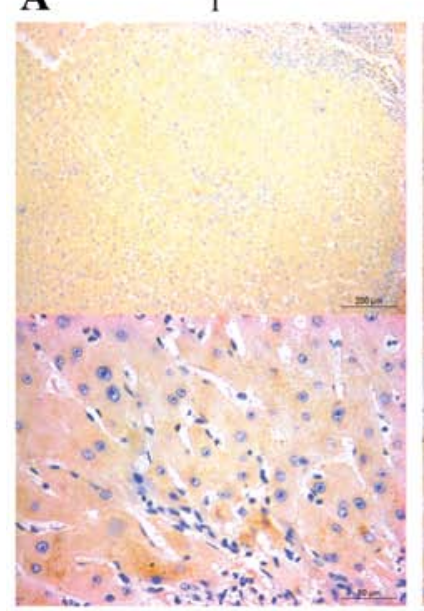

II

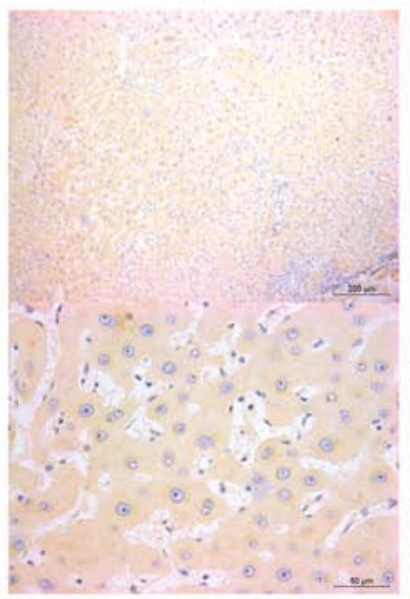

III

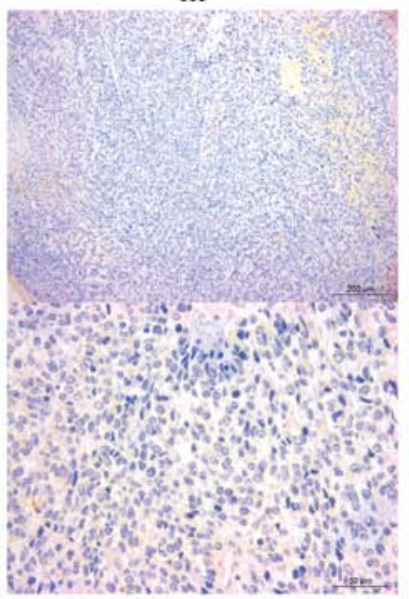

IV

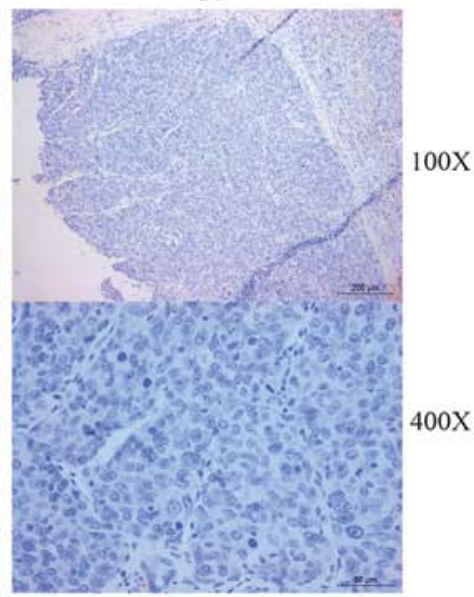

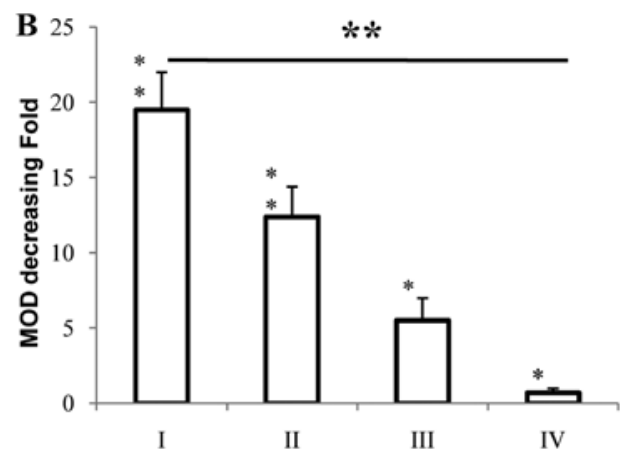

Figure 4. Expression of SMG-1 in HCC cases of different TNM stages. (A) Representative IHC analyses of SMG-1 expression in HCC tissues of different TNM stages. (B) Statistical quantification of the average MOD values of SMG-1 staining in HCC specimens of different TNM stages. The mean absorbance (MOD) of SMG-1 staining was decreased as HCC progressed to a higher clinical stage. ${ }^{*} \mathrm{P}<0.05$. 
Table III. Cox multivariate analyses of prognostic factors on overall survival.

\begin{tabular}{lcccr}
\hline Variable & $\beta$ & SE & Hazard ratio (95\% CI) & P-value \\
\hline AFP & 0.512 & 0.185 & $1.699(1.161-2.400)$ & 0.060 \\
Tumor size & 0.696 & 0.198 & $2.005(1.361-2.953)$ & 0.000 \\
Tumor multiplicity & 0.915 & 0.179 & $2.497(1.757-3.548)$ & 0.000 \\
Differentiation & 0.057 & 0.180 & $1.059(0.744-1.507)$ & 0.752 \\
Stage & 0.126 & 0.216 & $1.134(0.743-1.731)$ & 0.560 \\
SMG-1 & 1.494 & 0.254 & $4.457(2.701-7.325)$ & 0.000 \\
\hline
\end{tabular}

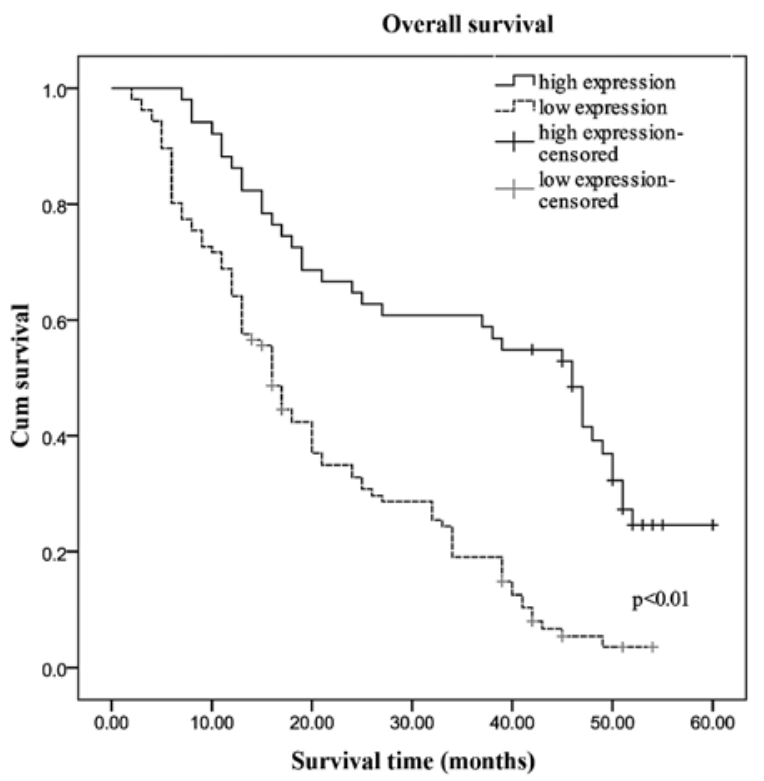

Figure 5. Low SMG-1 expression is associated with poor overall survival as analyzed by Kaplan-Meier survival analysis (log-rank test). ${ }^{* *} \mathrm{P}<0.01$.

Correlation of SMG-1 expression with overall survival of hepatocellular carcinoma patients. To determine the prognostic impact of SMG-1 on survival of postsurgical HCC patients, Kaplan-Meier survival analysis was performed. All of the 157 patients were followed up for survival until August 2013, and their survival data were stratified according to SMG-1 expression. Of the 157 patients, the mean survival period was 20.583 months for the patients with low SMG-1 expression, whereas it was 37.755 months for patients with high levels of SMG-1 expression $(\mathrm{P}<0.01)$. As shown in Fig. 5, Kaplan-Meier and log-rank survival tests indicated that low and high SMG-1 expression in HCC patients were indicative of different survival times; the OS of patients expressing high SMG-1 in their HCC lesions was much longer that the OS of the cases with low SMG-1 expression $(\mathrm{P}<0.01)$.

The prognostic effect of SMG-1 was further confirmed by stratified survival analysis. Results showed that patients with low SMG-1 expression had a significantly reduced survival following surgical resection in 5 subgroups of HCC patients (Fig. 6). Expression of SMG-1 was correlated with OS in subgroups of HCC patients. Kaplan-Meier survival analyses were performed in subgroups according to factors that are attributed to the outcome of HCC patients (log-rank test). This supports the usefulness of SMG-1 protein as a new prognostic marker for HCC patients.

Univariate and multivariate analyses of prognostic variables in the hepatocellular carcinoma patients. To reveal variables with potential prognostic significance in HCC patients, univariate analyses (Kaplan-Meier survival analyses) for each variable were performed. SMG-1, as well as tumor size, serum AFP level, tumor multiplicity, clinical stage and tumor differentiation were shown to be responsible for the outcome of HCC patients (Table II). SMG-1, as well as tumor size, serum AFP level, tumor multiplicity, clinical stage and tumor differentiation were all significant prognostic indicators for OS of HCC patients.

According to the results of the multivariate analysis of these factors, the predictive ability of SMG-1 expression, tumor size and tumor multiplicity was confirmed (Table III). However, there were no significant associations between prognosis and the other clinicopathological features.

\section{Discussion}

SMG-1 participates in the regulation of normal cell growth and TNF $\alpha$-induced apoptosis (18), similar to other members of the PIKK family such as ATM (ataxia telangiectagia mutated) and ATR (ATM- and Rad3-related) (19), and has been shown to control G1/S cell cycle arrest (16). The signal pathways involved in regulation of the cell cycle from the G1-phase to the $\mathrm{S}$ phase are frequent targets of carcinogenic events. Thus, loss of SMG-1 may result in a general alteration in the homeostasis of normal cells due to increased survival potential, which may subsequently lead to tumorigenesis. A recent study demonstrated that SMG-1 is a tumor-suppressor gene which suppresses tumor growth via the regulation of both the p53 and Cdc25A signaling pathways (16). However, the role of SMG-1 in the development of primary hepatocellular carcinoma has not been reported.

In the present study, we analyzed the expression of SMG-1 mRNA and protein in HCC patients using real-time PCR assay and immunohistochemistry. The results derived from the different assays strongly suggest a correlation between the SMG-1 level and the clinical outcome of HCC. We found that the combination of SMG-1 mRNA and protein in HCC tissue specimens was significantly lower than that in the distant normal tissues. The abnormal expression of SMG-1 
A

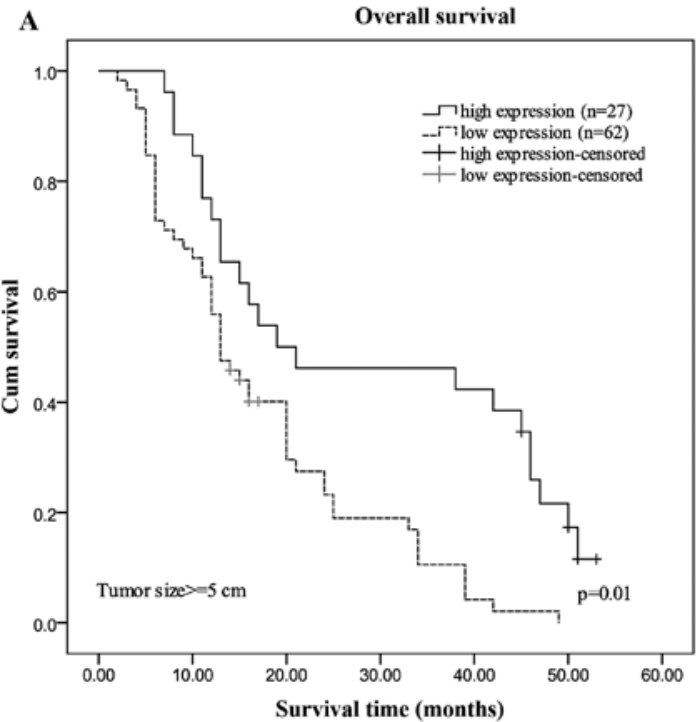

B

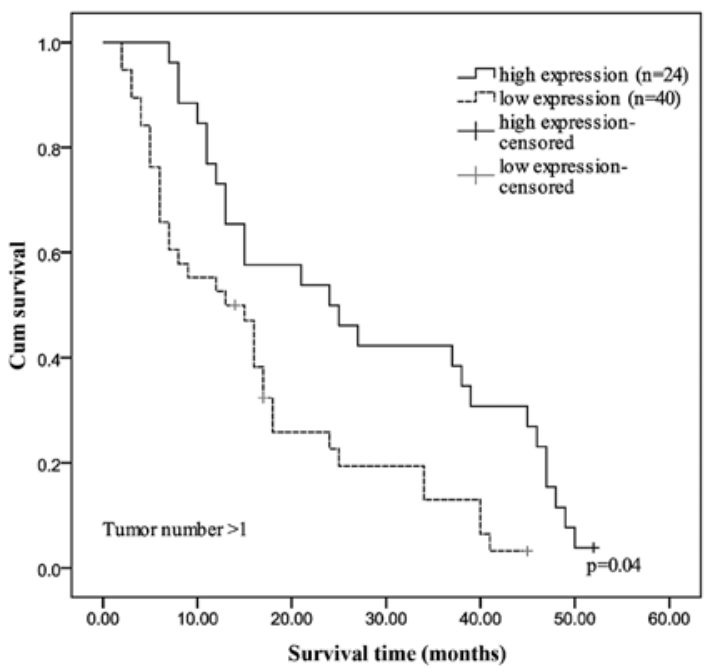

C

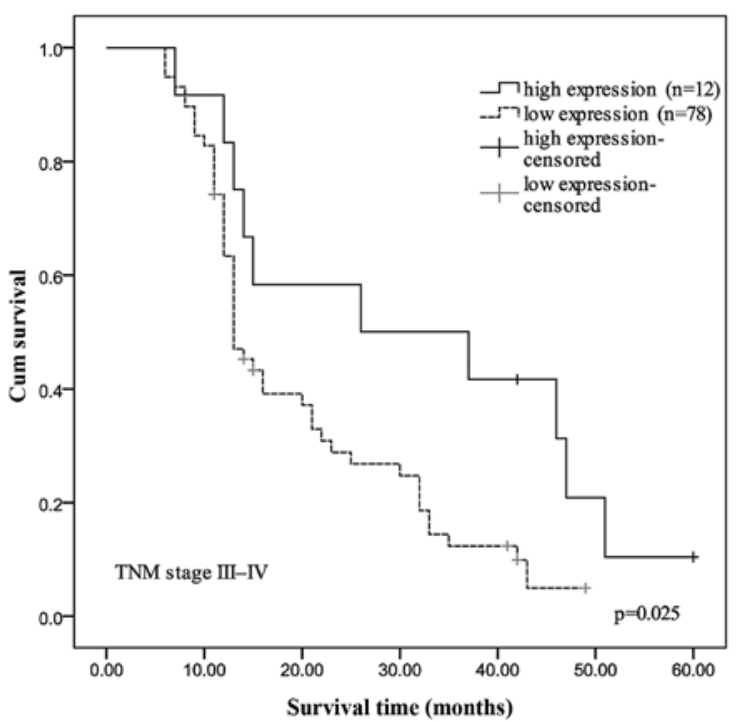

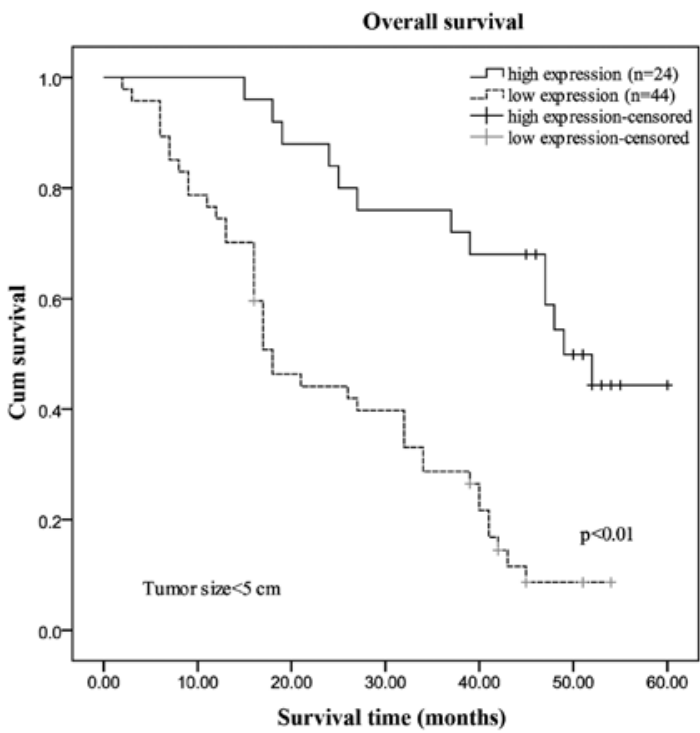

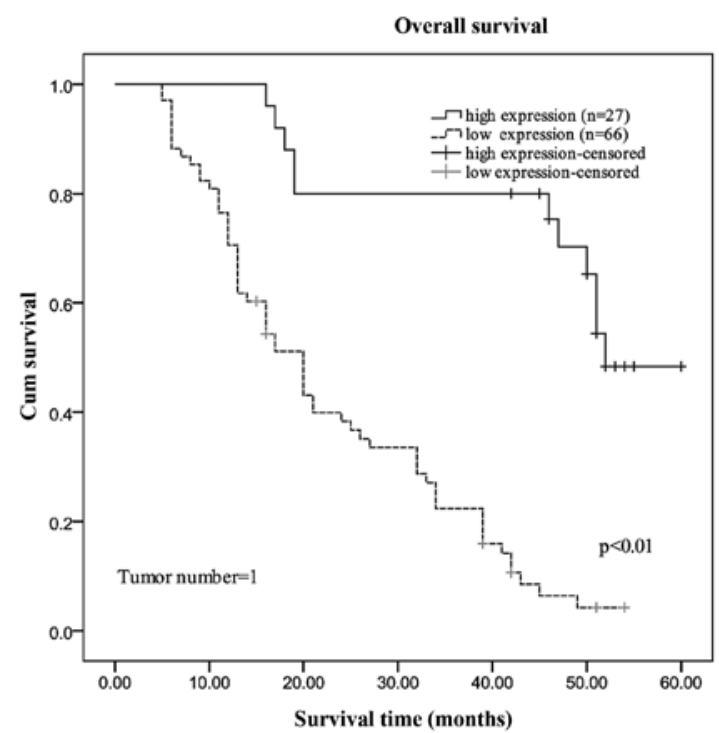

Overall survival

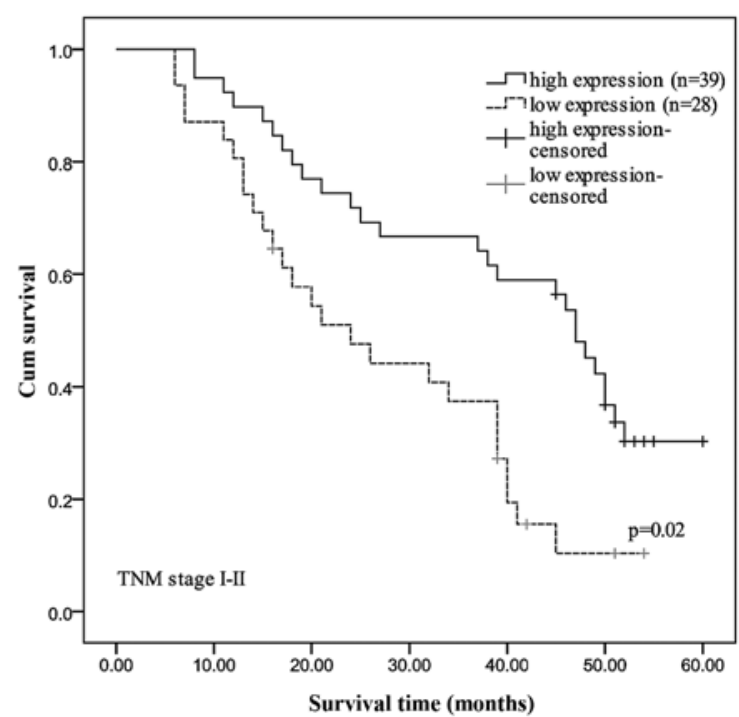

Figure 6. Kaplan-Meier analysis of the correlation of OS and SMG-1 expression in 157 patients stratified into HCC clinical subgroups. (A) Statistical significance of the difference between SMG-1 high-expressing and low-expressing patients was compared in subgroups of patients with large HCC lesions (diameter $\geq 5 \mathrm{~cm}$, left panel) and small HCC lesions (diameter $<5 \mathrm{~cm}$, right panel). (B) OS in patients with multitumor lesions (left panel) and a single tumor lesion (right panel). (C) OS rates in patients subgrouped into TNM stages III-IV (left panel) and TNM stages I-II (right panel). 

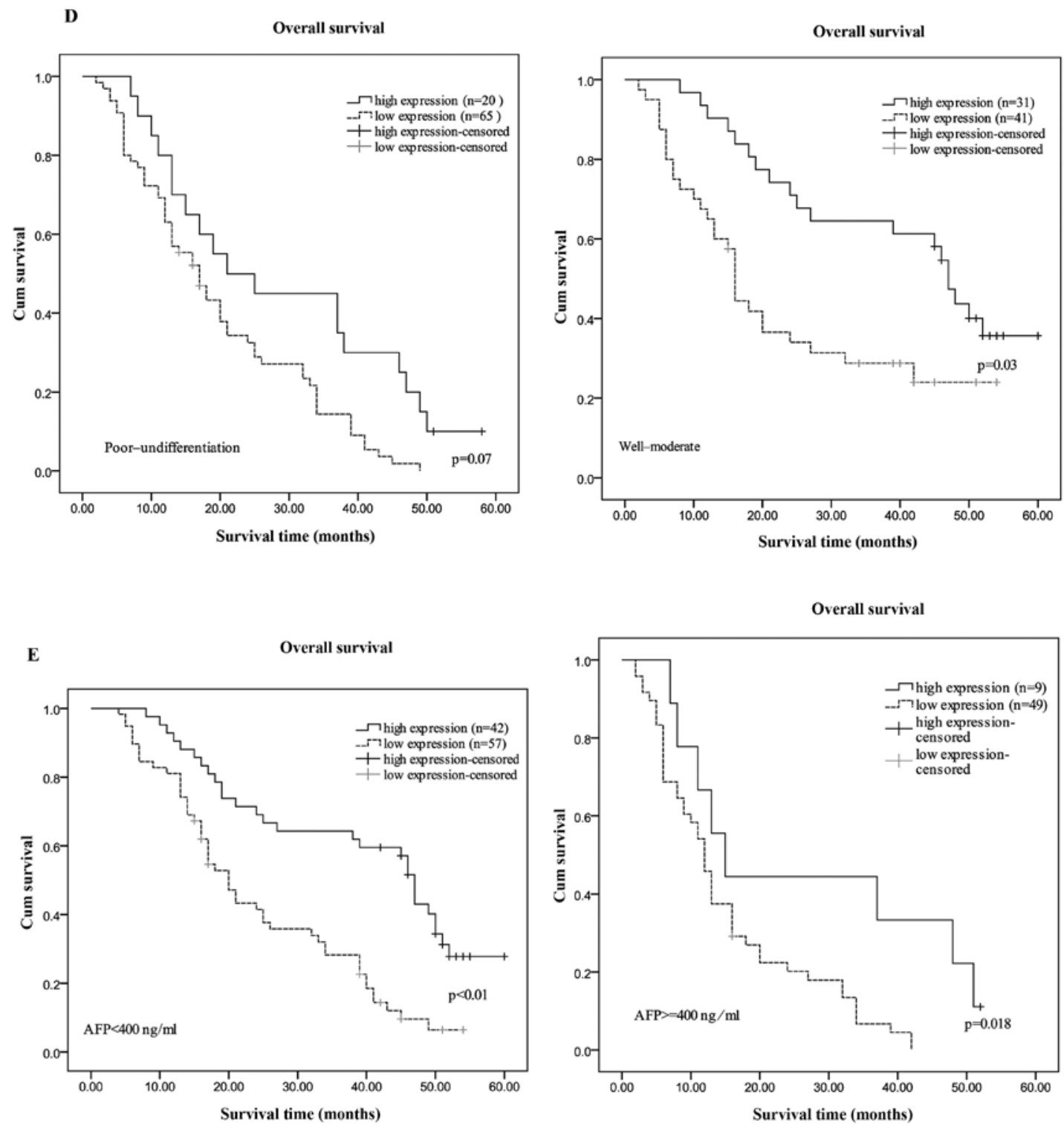

Figure 6. Continued. (D) OS rates in patients subgrouped according to HCC tumors with poor differentiation-undifferentiation (left panel) and well-moderate differentiation (right panel). (E) OS rates in patients subgrouped according to AFP $<400 \mathrm{ng} / \mathrm{ml}$ (left panel) and AFP $\geq 400 \mathrm{ng} / \mathrm{ml}$ (right panel) as differentiated by high or low SMG-1 expression.

associated with HCC was also correlated with higher AFP levels, poor differentiation and advanced TNM stages of HCC. Multivariate analysis confirmed that tumor size, tumor multiplicity and low expression of SMG-1 were risk factors for poor survival of HCC patients. The present study suggests that SMG-1, as a prognostic biomarker, is abnormally expressed in primary HCC tissues and is related to tumorigenesis, and detection of SMG-1 may provide new opportunities for prediction of HCC patient survival.

It is known that SMG-1 inhibits the mTOR (mechanistic target of rapamycin) signaling pathway which contributes to the regulatation of cell growth, metabolism and aging in response to nutrients, cellular energy stage and growth factors, thus, inhibiting the development of cancer and other diseases of aging (15). Numerous studies have demonstrated the importance of the mTOR pathway in patients with HCC (20-22). More importantly, it has also been suggested that the mTOR pathway is associated with the poor prognosis of $\operatorname{HCC}(23,24)$. Most of the genes which inhibit the mTOR pathway have been confirmed as tumor-suppressor genes in HCC. Furthermore, the mTOR inhibitor has been used as a potential target for the prevention and treatment of HCC (25). This implies that SMG-1, which can inhibit the mTOR signaling pathway, may be a new tumor-suppressor gene in primary HCC. The present study found that SMG-1 was deleted or weakly expressed in primary HCC tissues. Although the process by which SMG-1 functions to suppress $\mathrm{HCC}$ development or progression is still unclear, we believe that SMG-1 may contribute to inhibition of the tumorigenesis and development of $\mathrm{HCC}$ by negatively regulating the mTOR pathway. The mTOR pathway is more significantly altered in poorly differentiated and high-TNM stage tumors, and tumors with poor prognostic features $(23,24)$. In the present study, low SMG-1 expression was more frequently observed in poorly differentiated and high-TNM 
stage tumors. These findings can be explained by the fact that decreased or deleted expression of SMG-1 results in the loss of the negative regulation of the mTOR signaling pathway, resulting in the exacerbation of $\mathrm{HCC}$. Yet, further research is needed to fully understand the underlying molecular mechanisms.

Due to their critical role in the regulation of the cell cycle and cell growth, DNA damage restructuring and maintaining telomere length, PIKK family proteins, including PI3K, PI4K, TEL1, RAD3, ATM and ATR, have been attracting increased attention in regards to their significance in human cancers. Notably, altered expression of PIKKs commonly have a significant prognostic value in cancer. Lee et al (26) reported that aberrant expression of ATM plays a critical role in the development and progression of gastric cancer. Expression of PI3K was significantly associated with aggressive behavior and shorter DSS in non-gastrointestinal stromal soft tissue sarcomas (27). Bhattacharya et al (28) showed that inactivation of the ATM pathway may have an important role in the development of breast carcinoma with diagnostic, prognostic and therapeutic implications. Similar findings in other studies have found that expression of ATM may be a useful marker with which to identify patients presenting with pancreatic neuroendocrine tumors with poor prognosis who may benefit from close follow-up and aggressive therapy (29), and may be a possible prognostic factor in Chinese patients with CLL (30). Our findings suggest that SMG-1 also has similar functions, and can be used as an independent prognostic factor for the OS of HCC patients. In the present study, a decrease in SMG-1 was associated with poor prognosis of the HCC patients. Numerous independent studies have shown that the high potential for vascular invasion and metastasis is often the main biological basis for the poor prognosis of $\operatorname{HCC}(31,32)$. $\mathrm{p} 53$, one of the key players in the processes of cell dissociation, angiogenesis and cell migration in $\operatorname{HCC}(33,34)$, has been shown to be regulated by SMG-1 (16). This suggests that expression of SMG-1 may be associated with the prognosis of $\mathrm{HCC}$ via regulation of the expression of P53, which is consistent with our results.

It is widely recognized that early diagnosis and treatment are crucially needed for a more favorable prognosis of HCC patients. Using ideal biomarkers to identify patients with a higher risk of a worse prognosis may reduce the mortality rate of HCC patients. In our study, the patients with early-stage HCC (TNM stages I-II) also displayed a significantly lower level of SMG-1 in HCC lesions than that in the normal liver tissues. In addition, as HCC progressed to later stages, the SMG-1 level further decreased. Thus, these characteristics of SMG-1 further confirm that SMG-1 may be a significant biomarker for identifying patients with poor prognosis after surgical intervention in early disease stages.

In summary, the present study demonstrated that SMG-1 was frequently deleted or downregulated in HCC. Abnormal expression of SMG-1 was significantly correlated with differentiation, clinical stage and serum AFP level, suggesting that SMG-1 may be involved in the pathogenesis and development of HCC. Low SMG-1 expression adversely impacted the survival of HCC patients. Consequently, our data revealed that low SMG-1 expression plays a crucial role in predicting the postsurgical overall survival of HCC patients.

\section{Acknowledgements}

The authors thank Professor Chen Huang of Xi'an Jiaotong University (Xi'an, China) for providing the experimental platform and expert opinions.

\section{References}

1. Jemal A, Bray F, Center MM, Ferlay J, Ward E and Forman D: Global cancer statistics. CA Cancer J Clin 61: 69-90, 2011.

2. He G and Karin M: NF- $\mathrm{BB}$ and STAT3 - key players in liver inflammation and cancer. Cell Res 21: 159-168, 2011.

3. Chen L, Ho DW, Lee NP, Sun S, Lam B, Wong KF, Yi X, Lau GK, Ng EW, Poon TC, Lai PB, Cai Z, Peng J, Leng X, Poon RT and Luk JM: Enhanced detection of early hepatocellular carcinoma by serum SELDI-TOF proteomic signature combined with alphafetoprotein marker. Ann Surg Oncol 17: 2518-2525, 2010.

4. Sidransky D: Emerging molecular markers of cancer. Nat Rev Cancer 3: 210-219, 2002.

5. El-Serag HB and Rudolph KL: Hepatocellular carcinoma: Epidemiology and molecular carcinogenesis. Gastroenterology 132: 2557-2576, 2007.

6. Zhao Y, Wang WJ, Guan S, Li HL, Xu RC, Wu JB, Liu JS, Li HP, Bai W, Yin ZX, Fan DM, Zhangand ZL and Han GH: Sorafenib combined with transurethral chemoembolization for the treatment of advanced hepatocellular carcinoma: a largescale multicenter study of 222 patients. Ann Oncol 7: 1786-1792, 2013.

7. Denning G, Jamieson L, Maquat L, Thompson E and Fields A: Cloning of a novel phosphatidylinositol kinase-related kinase: characterization of the human SMG-1 RNA surveillance protein. J Biol Chem 276: 22709-22714, 2001.

8. Yamashita A, Ohnishi T, Kashima I, Taya Y and Ohno S: Human SMG-1, a novel phosphatidylinositol 3-kinase-related protein kinase, associates with components of the mRNA surveillance complex and is involved in the regulation of nonsense-mediated mRNA decay. Genes Dev 15: 2215-2228, 2001.

9. Grimson A, O'Connor S, Newman CL and Anderson P: SMG-1 is phosphatidylinositol kinase-related protein kinase required for nonsense-mediated mRNA decay in Caenorhabditis elegans. Mol Cell Biol 24: 7483-7490, 2004.

10. Gehen S, Staversky R, Bambara R, Keng P and O'Reilly M: SMG-1 and ATM sequentially and independently regulate the G1 checkpoint during oxidative stress. Oncogene 27: 4065-4074, 2008.

11. Cheung H, St Jean M, Beug S, Lejmi-Mrad R, LaCasse E, Baird S, Stojdl D,Screaton R and Korneluk R: SMG1 and NIK regulate apoptosis induced by Smac mimetic compounds. Cell Death Dis 2: e146, 2011.

12. Brumbaugh K, Otterness D, Geisen C, Oliveira V, Brognard J, Li X, Lejeune F, Tibbetts R, Maquat L and Abraham R: The mRNA surveillance protein hSMG-1 functions in genotoxic stress response pathways in mammalian cells. Mol Cell 14: 585-598, 2004.

13. Chen R, Yang Q, Chen Y, Oliveira V, Dalton W, Fearns C and Lee J: Kinome siRNA screen identifies SMG-1 as a negative regulator of hypoxia-inducible factor- $1 \alpha$ in hypoxia. J Biol Chem 284: 16752-16758, 2009.

14. Masse I, Molin L, Mouchiroud L, Vanhems P, Palladino F, Billaud $M$ and Solari F: A novel role for the SMG-1 kinase in lifespan and oxidative stress resistance in Caenorhabditis elegans. PLoS One 3: e3354, 2008.

15. González-Estévez C, Felix DA, Smith MD, Paps J, Morley SJ, James V, Sharp TV and Aboobaker AA: SMG-1 and mTORC1 act antagonistically to regulate response to injury and growth in planarians. PLoS Genet 3: e1002619, 2012.

16. Gubanova E, Issaeva N, Gokturk C, Djureinovic T and Helleday T: SMG-1 suppresses CDK2 and tumor growth by regulating both the p53 and Cdc25A signaling pathways. Cell Cycle 12: 3770-3780, 2013.

17. Roberts T, Ho U, Luff J, Lee C, Apte S, MacDonald K, Raggat L, Pettit A, Morrow C and Waters M: Smg1 haploinsufficiency predisposes to tumor formation and inflammation. Proc Natl Acad Sci USA 110: E285-E294, 2013.

18. Oliveira V, Romanow WJ, Geisen C, Otterness DM, Mercurio F, Wang HG, Dalton WS and Abraham RT: A protective role for the human SMG-1 kinase against tumor necrosis factor- $\alpha$-induced apoptosis. J Biol Chem 283: 13174-13184, 2008. 
19. Shiloh Y: ATM and related protein kinases: Safeguarding genome integrity. Nat Rev Cancer 3: 155-168, 2003.

20. Matter MS, Decaens T, Andersen JB and Thorgeirsson SS Targeting the mTOR pathway in hepatocellular carcinoma: current state and future trends. J Hepatol 60: 855-865 2014

21. Menon S, Yecies JL and Zhang HH: Chronic activation of mTOR complex 1 is sufficient to cause hepatocellular carcinoma in mice. Sci Signal 5: ra24, 2012.

22. Bassullu N, Turkmen I, Dayangac M, Yagiz Korkmaz P, Yasar R, Akyildiz M, Yaprak O, Tokat Y, Yuzer Y and Bulbul Dogusoy G: The predictive and prognostic significance of c-erb-B2, EGFR, PTEN, mTOR, PI3K, p27, and ERCC1 expression in hepatocellular carcinoma. Hepat Mon 12: e7492, 2012.

23. Villanueva A, Chiang DY, Newell P, Peix J, Thung S, Alsinet C, Tovar V, Roayaie S, Minguez B, Sole M, Battiston C, Van Laarhoven S, Fiel MI, Di Feo A, Hoshida Y, Yea S, Toffanin S, Ramos A, Martignetti JA, Mazzaferro V, Bruix J, Waxman S, Schwartz M, Meyerson M, Friedman SL and Llovet JM: Pivotal role of mTOR signaling in hepatocellular carcinoma. Gastroenterology 135: 1972-1983.e1-11, 2008.

24. Zhou L, Huang Y, Li J and Wang Z: The mTOR pathway is associated with the poor prognosis of human hepatocellular carcinoma. Med Oncol 27: 255-261, 2010.

25. Buitrago-Molina LE and Vogel A: mTor as a potential target for the prevention and treatment of hepatocellular carcinoma. Curr Cancer Drug Targets 9: 1045-1061, 2012.

26. Lee HE, Han N, Kim MA, Lee HS, Yang HK, Lee BL and Kim WH: DNA damage response-related proteins in gastric cancer: ATM, Chk2 and p53 expression and their prognostic value. Pathobiology 1: 25-35, 2014.

27. Valkov A, Kilvaer TK, Sorbye SW, Donnem T, Smeland E, Bremnes RM and Busund LT: The prognostic impact of Akt isoforms, PI3K and PTEN related to female steroid hormone receptors in soft tissue sarcomas. J Transl Med 9: 200, 2011.
28. Bhattacharya N, Mukherjee N, Singh RK, Sinha S, Alam N, Roy A, Roychoudhury S and Panda CK: Frequent alterations of MCPH1 and ATM are associated with primary breast carcinoma: clinical and prognostic implications. Ann Surg Oncol 3: 424-432, 2013.

29. Shin JU, Lee CH, Lee KT, Lee JK, Lee KH, Kim KM, Kim KM, Park SM and Rhee JC: Prognostic significance of ATM and cyclin B1 in pancreatic neuroendocrine tumor. Tumour Biol 5 1645-1651, 2012.

30. Xu W, Li JY, Wu YJ, Yu H, Shen QD, Li L, Fan L and Qiu HX Prognostic significance of ATM and TP53 deletions in Chinese patients with chronic lymphocytic leukemia. Leuk Res 7: 1071-1077, 2008.

31. Llovet JM and Bruix J: Novel advancements in the management of hepatocellular carcinoma in 2008. J Hepatol 48 (Suppl 1): S20-S37, 2008.

32. Bruix J, Sherman M, Llovet JM, Beaugrand M and Lencioni R: Clinical management of hepatocellular carcinoma. Conclusions of the Barcelona-2000 EASL conference. European Association for the Study of the Liver. J Hepatol 35: 421-430, 2001.

33. Tseng PL, Tai MH, Huang CC, Wang CC, Lin JW, Hung CH, Chen $\mathrm{CH}$, Wang JH, Lu SN, Lee CM, Changchien CS and $\mathrm{Hu}$ TH: Overexpression of VEGF is associated with positive p53 immunostaining in hepatocellular carcinoma (HCC) and adverse outcome of HCC patients. J Surg Oncol 5: 349-357, 2008.

34. Hsu H, Peng S, Lai P, Chu J and Lee P: Mutations of p53 gene in hepatocellular carcinoma correlate with tumor progression and patient prognosis: a study of 138 patients with unifocal HCC. Int J Oncol 6: 1341-1347, 1994 